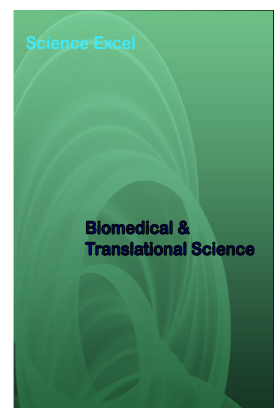

Correspondence

Marjan Assefi

University of North Carolina, Greensboro, North Carolina, USA

E-mail: massefi@aggies.ncat.edu

- Received Date: 14 July 2021

- Accepted Date: 19 July 2021

- Publication Date: 28 July 2021
Copyright

(C) 2021 Science Excel. This is an openaccess article distributed under the terms of the Creative Commons Attribution 4.0 International license.

\title{
Association between the rs 8028440 polymorphism of CYFIP 1 gene in autism patients
}

\author{
Hossein Ghahramani Almanghadim ${ }^{1,2}$, Marjan Assefi ${ }^{1,2^{*}}$, Shahab Masoumi ${ }^{3}$, \\ Parisa Vakili ${ }^{3}$, Zinat Shams ${ }^{4}$, Atefeh Moradkhani ${ }^{5}$, Mahmoud Shekari \\ Khaniani ${ }^{6}$, Sima Mansouri Derakhshan ${ }^{6}$
}

\author{
'Department of Biological Science, Ahar Branch, Islamic Azad University, Ahar, IR Iran \\ ${ }^{2}$ University of North Carolina at Greensboro, USA \\ ${ }^{3}$ Department of Administrative Science, Vancouver Campus, Fairleigh Dickinson University (FDU), Vancouver, British Columbia, \\ Canada, University of Berkeley, USA \\ ${ }^{4}$ Biochemistry, Department of Biological Science, Kharazmi University Tehran, Iran \\ ${ }^{5}$ Department of Biology, Faculty of Science, Zanjan Branch, Islamic Azad University, Iran \\ ${ }^{6}$ Department of Medical Genetics, Faculty of Medicine, Tabriz University of Medical Sciences, Tabriz, Iran
}

\begin{abstract}
Demonstrated no association between ASD and rs 8028440 polymorphism of the CYFIP1 gene, which needs further studies in a larger population of ASD subjects to find the contribution of rs 8028440 polymorphism in CYFIP1 gene with ASD in Iranian patients.

Introduction: Given the importance of the Cytoplasmic FMR1 Interacting Protein 1 (CYFIP1 gene) in relation to neurodevelopmental abnormalities such as autism spectrum disorder (ASD), recognizing the interaction between single nucleotide polymorphisms (SNPs) of this gene in autism cases is important. In this study, we evaluated the probable association of rs 8028440 polymorphism of the CYFIP1 gene with ASD disorder in Iranian subjects.

Methods and patients: The CYFIP1 gene were amplified with specific primers and the PCR products were digested with RsaI restriction enzyme to obtain the rs 8028440 polymorphism in 100 ASD patients and 100 healthy control cases. Finally, the samples were genotyped using direct sequencing to identify $\mathrm{CC}, \mathrm{CT}$, and TT genotypes.

Results: The Hardy-Weinberg equilibrium showed no significant deviation in the subjected population. According to our results, the frequency of the $\mathrm{C}$ allele was higher in ASD groups than in the control group. The full length of PCR was $662 \mathrm{bp}$ and through RFLP-PCR, normal genotype (C/C), heterozygote genotype $(T / C)$, and homozygous genotype $(T / T)$ was detected. Ten PCR products were sequenced and the corresponded alleles $\mathrm{A} / \mathrm{T}$ and $\mathrm{Y}(\mathrm{C}$ or $\mathrm{T})$ were determined. It was revealed no significant difference was found between ASD subjects and controls with respect to the frequency of the rs 8028440 gene allele.
\end{abstract}

\section{Introduction}

Autism spectrum disorders (ASDs) (OMIM 209850), are a heterogeneous group of pervasive neurodevelopmental disorders characterized by severe impairments in reciprocal social communications, repetitive paradigms of behavior, restricted range of interests, and stereotyped interests and activities [1]. Family-based genetic and twin studies of ASD have demonstrated high heritability index (0.89-0.92) with a complex multifactorial etiology and the phenotype variability of ASD could be explained by both genetics and environmental factors [2-6]. Furthermore, meta-analytical studies of twins revealed that monozygotic twins represent a considerably higher concordance rate of ASD compared with dizygotic twins stressing the importance of genetic factors in ASD [7,8]. Several lines of evidence have introduced a variety of genes that are robustly considered to be involved with increased risk of ASDs. Among the genes, Gamma-Aminobutyric Acid Type A Receptor Subunit Alpha2 (GABRA2), GABRA4, Gamma-Aminobutyric Acid Type A Receptor Subunit Betal (GABRB1), GABRB2, GABRB3, Tryptophan 2,3-Dioxygenase (TDO2), Calcium-binding mitochondrial carrier protein coding SLC25A12, and brainderived neurotrophic factor (BDNF) have been commonly used for ASD examination [9-11]. Further genome sequencing have led to the identification of several locus including AUTS1 to AUTS 18, CNTNAP2, SHANK3, CHD8, KIA044 and signaling proteins such as Rho family GTPases that are extensively associated with ASD [12]. Rho family GTPases are a group of small $G$ proteins that acting as key regulators of the actin cytoskeleton transducing the upstream 
signals to downstream effectors in a plethora of cellular processes like cell growth, differentiation, adhesion, and migration. Rho GTPases proved to have an essential role in various neuronal morphology and disfunction particularly ASD [13-16]. Moreover, according to the ASD-related genes databases e.g., Simon's foundation autism research initiative (SFARI), RhoGEFs, RhoGAPs, and Rho effectors have been suggested as ASD risk genes. These genes are directly participated in signaling pathways mediated by Rho GTPases and encode three major categories of regulatory proteins including Ras homolog family member A (RhoA), Ras-related C3 Botulinum Toxin Substrate 1 (Rac1), and cell division cycle 42 (Cdc42). Accordingly, Cytoplasmic FMR1 Interacting Protein (CYFIP1) as a Rho GTPase E effector and its association with neural evolutionary abnormalities have gained a great amount of attention [17]. CYFIP1 is a Rac1-interacting protein coded by CYFIP1 gene located at $15 \mathrm{q} 11-\mathrm{q} 13$ and is identified to be contributed with the WAVE regulatory complex which regulates actin filament associated with autism $[18,19]$. Several studies have revealed that patients with neurodevelopmental disease including ASD, attentiondeficit/hyperactivity disorder (ADHD), internal disability (ID), schizophrenia (SCZ), and obsessive-compulsive disorder (OCD) microdeletions, represent a microdeletions at 15 q11.2 and microduplications between breakpoints first and second breakpoints [20-22]. In a study of patients with Highfunctioning autism (HFA), several rare variants of paternally inherited CYFIP1 have been identified in autistic patients with a de novo SHANK2 deletion [23,24]. Additionally, single nucleotide polymorphisms (SNPs) in CYFIP1 were stated to be correlated with ASD in a couple of studies $[25,26]$. An investigation on the expression of CYFIP1 mRNA in the human dorsolateral prefrontal cortex showed increased levels of CYFIP1 expression in ASD and classical autism patients along with identification of several common variants of CYFIP1 in these patients [27]. In a recent investigation, a substantial increase in CYFIP1 transcript levels were recognized by examination of peripheral blood samples of ASD subjects [28]. All these clinical outcomes have revealed that the altered levels of CYFIP1 could contribute the pathology of ASD. CYFP1 is broadly expressed in multiple cells except liver cells during tissue development with an enhanced expression level in cerebral cortex, hippocampus, olfactory bulb, cerebellum, and lateral septum in the adult brain. The mentioned features describe CYFIP 1 as an autism associated gene with increased expression levels in the patients. Given the importance of the CYFIP1 gene in neurodevelopmental abnormalities such as ASD, providing an effective recognition method for high throughput detection of CYFIP1 polymorphism that plays an important role in early detection of ASD. Transmission disequilibrium test and odds ratios analysis have identified several SNPs including rs8028440, rs2289823, rs7403800, and rs3751566 of CYFIP1, which are associated with ASD. In this regard, the main purpose of presented study was to compare the incidence of rs 8028440 polymorphism in autism patients.

\section{Patients and methods}

\section{Subjects}

This study was a case-control investigation and was approved by the Regional Ethics Committee of Tabriz University of Medical Sciences (Tabriz, Iran) by the number of 92/2-2.2. The number of samples was determined using the following Cochran formula (Equation 1):

$$
n=\frac{z^{2} p(1-p)}{e^{z}}
$$

Patients were diagnosed according to the Diagnostic and Statistical Manual of Mental Disorders (DSM-IV, fifth edition), and accurate taking of the patient's history and psychological condition and then was introduced to the Avicenna Medical Genetic Laboratory (Tabriz, Iran). Control subjects were chosen from the general population with no psychiatric abnormality backgrounds based upon questionnaire responses and self-reporting. Two hundred of ASD subjects (aged under 15 years) were selected based on the inclusion and exclusion criteria and after signing the informed consent were entered in the examination. Fresh peripheral blood (5-3 ml) was obtained from subjects and was transferred to the falcon tubes $(15 \mathrm{ml})$ containing $0.5 \mathrm{ml}$ anticoagulant EDTA $(0.5 \mathrm{M})$ and the samples were maintained at $-20^{\circ} \mathrm{C}$. The subjects with other neurological and psychosocial disease such as fragile $\mathrm{X}$ syndrome were excluded from the study.

\section{Genotyping}

Genomic DNA was extracted from peripheral whole blood based on the salting-out principle [29]. The purity and yield of the extracted DNA were verified regarding the absorbance at $\mathrm{A}_{260} / \mathrm{A}_{280}$ ratio. Subsequently, polymerase chain reaction (PCR) was performed in a final volume of 25 $\mu \mathrm{L}$ that contain $\sim 100 \mathrm{ng}$ of genomic DNA, $4 \mathrm{pmol} / \mathrm{l}$ of each primer (forward: $5^{\prime}$ - TCACCTCACTCTGTCGCTGG $-3^{\prime}$ and reverse: 5 ' - CCAGTGCTTGCAGACTTCTTTC -3'), $2.5 \mu \mathrm{L} 10 \mathrm{x}$ PCR buffer, $0.25 \mathrm{mM}$ of each dNTP, $1.5 \mathrm{mM} \mathrm{MgCl}, 2$ units of SmarTaq DNA polymerase (Cinnagen, Iran), and $2.5 \mu \mathrm{L}$ of Q solution. The profile of PCR cycling was as follows: an initial denaturation cycle at $95^{\circ} \mathrm{C}$ for 5 minutes; 35 cycles at $95^{\circ} \mathrm{C}$ for 45 seconds, $63^{\circ} \mathrm{C}$ for 30 seconds, $72^{\circ} \mathrm{C}$ for 1 minutes, and a final extension at $72^{\circ} \mathrm{C}$ for 10 minutes was performed using Gene Amp@ PCR System 9700 (Applied Biosystem).The PCR products were digested with restriction enzyme, Rsa I, followed by $17 \mathrm{~h}$ treatment at $37^{\circ} \mathrm{C}$ and the obtained fragments were visualized by $4 \%$ agarose gel electrophoresis. Finally, $10 \%$ of samples were randomly selected and subjected to Sanger sequencing for the detection of mutations.

\section{Statistical analyses}

Deviation from the Hardy-Weinberg equilibrium was checked through Fisher's exact test in the studied SNP. The difference between the genotype and allele frequencies was obtained by Pearson's $\chi^{2}$-tests. The $\mathrm{p}$-value $<0.05$ (twotailed) was statistically considered as a significant level. The distribution of genotype incidence was examined according to the genetic models (log-additive, over dominant, recessive, dominant, and Codominant) using the SNPassoc package of $\mathrm{R}$ version 3.2.0. Other statistical analyzes were carried out by means of $\mathrm{R}$ version 3.2.0. All statistical analyses were conducted through the Stata statistical package.

\section{Results}

The genotypic distributions of rs8028440 SNP examined in this study were in Hardy-Weinberg equilibrium $(\mathrm{P}>0.05$, Table 1) which confirmed no deviation in the subjected population. 
Table 1. Results of Hardy Weinberg equilibrium (HWE) using chi square test.

\begin{tabular}{|c|c|c|c|c|}
\hline HWE & All & Control & Case & P value \\
\hline rs8028440 & 0.229097 & 0.295662 & 0.748962 & 0.5 \\
\hline
\end{tabular}

Table 1. Genotype distribution and allele frequencies in autism and control groups

\begin{tabular}{|c|c|c|c|c|c|c|c|c|c|}
\hline \multirow{2}{*}{ Gene (SNP) } & \multirow{2}{*}{ Subjects } & \multicolumn{3}{|c|}{ Genotype frequencies (\%) } & \multirow{2}{*}{$\mathrm{p}$} & \multicolumn{2}{|c|}{ Allele frequencies $(\%)$} & \multirow{2}{*}{ OR $(95 \% \mathrm{CI})$} & \multirow{2}{*}{$\mathrm{p}$} \\
\hline & & $\mathrm{C} / \mathrm{C}$ & $\mathrm{C} / \mathrm{T}$ & $\mathrm{T} / \mathrm{T}$ & & $\mathrm{C}$ & $\mathrm{T}$ & & \\
\hline \multirow{2}{*}{$\begin{array}{c}\text { CYFIP1 } \\
\text { (rs8028440) }\end{array}$} & $\begin{array}{c}\text { Autism } \\
(\mathrm{N}=100)\end{array}$ & $66(66 \%)$ & $30(30 \%)$ & $4(4 \%)$ & \multirow{2}{*}{0.2366} & $81(81 \%)$ & $26(26 \%)$ & \multirow{2}{*}{$0.57(0.76-0.38)$} & \multirow{2}{*}{0.5} \\
\hline & $\begin{array}{c}\text { Control } \\
(\mathrm{N}=100)\end{array}$ & $57(57 \%)$ & $34(34 \%)$ & $9(9 \%)$ & & $79(79 \%)$ & $19(19 \%)$ & & \\
\hline
\end{tabular}

Table 3. Analysis of Genotype and allele distributions of rs8028440 of CYFIP1 gene in autism and control groups

\begin{tabular}{|c|c|c|c|c|c|c|c|c|}
\hline & Control & $\%$ & Case & $\%$ & OR & lower & upper & p-value \\
\hline \multicolumn{9}{|c|}{ Codominant } \\
\hline $\mathrm{C} / \mathrm{C}$ & 57 & 57 & 66 & 66 & 1 & & & 0.2366 \\
\hline $\mathrm{C} / \mathrm{T}$ & 34 & 34 & 30 & 30 & 0.76 & 0.42 & 1.4 & \\
\hline $\mathrm{T} / \mathrm{T}$ & 9 & 9 & 4 & 4 & 0.38 & 0.11 & 1.31 & \\
\hline \multicolumn{9}{|c|}{ Dominant } \\
\hline $\mathrm{C} / \mathrm{C}$ & 57 & 57 & 66 & 66 & 1 & & & 0.1905 \\
\hline $\mathrm{C} / \mathrm{T}-\mathrm{T} / \mathrm{T}$ & 43 & 43 & 34 & 34 & 0.68 & 0.39 & 1.21 & \\
\hline \multicolumn{9}{|l|}{ Recessive } \\
\hline $\mathrm{C} / \mathrm{C}-\mathrm{C} / \mathrm{T}$ & 91 & 91 & 96 & 96 & 1 & & & 0.1466 \\
\hline $\mathrm{T} / \mathrm{T}$ & 9 & 9 & 4 & 4 & 0.42 & 0.13 & 1.42 & \\
\hline \multicolumn{9}{|c|}{ Over dominant } \\
\hline $\mathrm{C} / \mathrm{C}-\mathrm{T} / \mathrm{T}$ & 66 & 66 & 70 & 70 & 1 & & & 0.5442 \\
\hline $\mathrm{C} / \mathrm{T}$ & 34 & 34 & 30 & 30 & 0.83 & 0.46 & 1.51 & \\
\hline \multicolumn{9}{|c|}{ log-Additive } \\
\hline $0,1,2$ & 100 & 50 & 100 & 50 & 0.69 & 0.43 & 1.09 & 0.106 \\
\hline
\end{tabular}

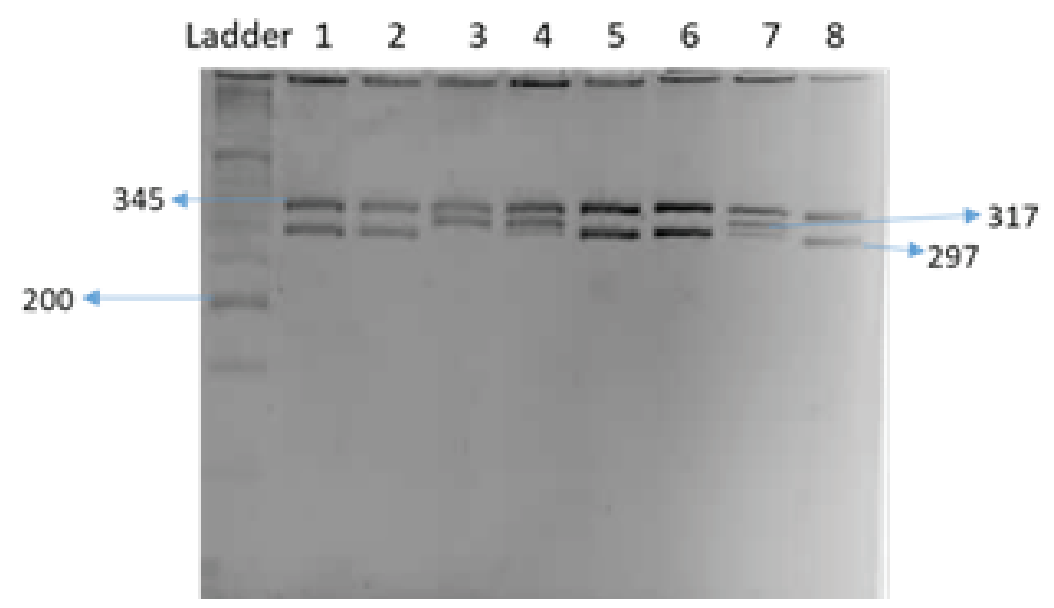

Figure 1. BsaI PCR-RFLP results of the rs 8028440 polymorphism. DNA ladder (50bp); Lane1, 2,5,6,8, are CC; Lane 3 is TT, and Lane 4, 7 are $G G$ genotypes. 
A

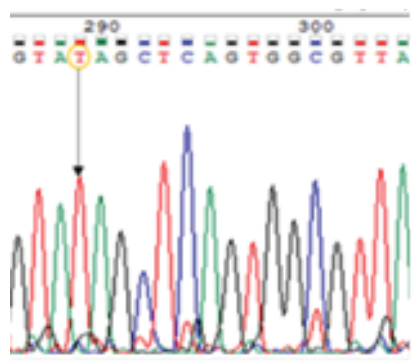

B

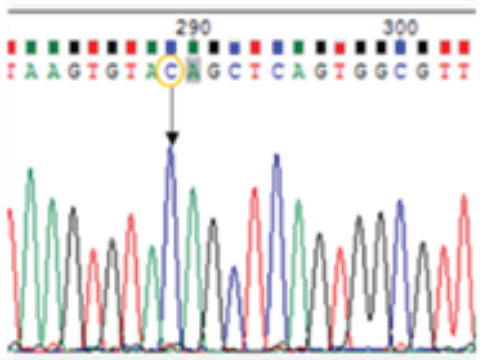

C

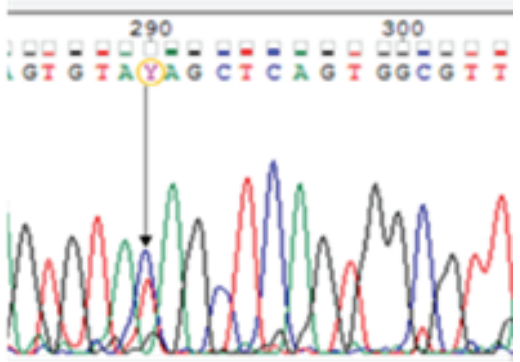

Figure 1. Examples of RFLP-PCR product of CYFIP1 gene. (A) TT genotype, (B) CC genotype and (C) T/C genotype for polymorphism of rs 8028440 .

The allele frequency of rs8028440 SNP in both control and autism subjects are explained in the Table 2. According to the results, frequency of the $\mathrm{C}$ allele was boosted in autism groups than control. The result of restriction enzymes on the $4 \%$ gel electrophoresis is demonstrated in Figure 1. The full length of PCR product was 662 base pairs (bp) and normal genotype $(\mathrm{C} / \mathrm{C})$, heterozygote genotype $(\mathrm{T} / \mathrm{C})$ and homozygous genotype (T/T) were detected through RFLP-PCR. After digestion with RsaI, homozygous allele C represented two bands (345 and $297 \mathrm{bp}$ ), the homozygous allele T presented two bands of 317 and $345 \mathrm{bp}$, and heterozygote genotype $\mathrm{C} / \mathrm{T}$ with three bands $(345,317,297 \mathrm{bp})$. Ten PCR products were further analysis of CYFIP1 with Sanger's sequencing method (Figure 2). The arrow corresponds to alleles A/ T and Y represents the polymorphism sites. Table 3 reveals no significant difference between autism subjects and control samples with respect to gene allele frequencies of rs8028440.

\section{Disscusion}

TASD is a genetically heterogenic neurodevelopmental disorder featured by deficits in social communication including restrictive and repetitive behaviors. Regarding the common symptoms and features of neurodevelopmental abnormalities, fast and early detection of ASD has become a major challenge. Hence, a demand for developing accurate bioanalytical approaches for further illumination of the ASD underlying molecular mechanism and pathways has been stressed. It has been reported that the $15 \mathrm{q} 11-13$ chromosomal region is mainly involved in most ASD abnormalities due to the presence of various chromosomal breakpoints and internal duplications. Duplications of these regions lead to cognitive and behavioral problems, limited speaking skills, and a higher propensity to develop severe ASD symptoms. A great amount of attention has been focused on a detailed analysis of the CYFIP1 gene due to its potential involvement in the etiology of ASD. Overexpression of CYFIP1 and $15 q$ duplication regulates the formation and functionalization of the dendritic spine in the wave regulatory complex during actin polymerizationb[30]. Consequently, the process affects the translation of synaptic proteins through interaction with fragile $\mathrm{X}$ mental retardation protein (FMRP). Indeed, dysregulation of ASD susceptibility genes such as mTOR signaling accounts for abnormal neuronal morphology and intellectual disabilities [31].

Based on the association of CYFIP1/2 and central nervous system disorders such as ASD, Norozi et al. in 2018 confirmed the significant overexpression of CYFIP1/2 in ASD patients in comparison with healthy subjects. They have demonstrated significant overexpression of the CYFIP1/2 gene in the ASD group compared with healthy subjects using Real-time PCR analysis. In addition, a correlation between age and ASD intensity was reported in this case. These results confirmed the role of CYFIP1/2 in the pathogenesis of ASD and its potential as an ASD diagnosis marker [28].

According to the genotyping data and mRNA expression analysis from the SNPs database, seven variants of to CYFIP1 alleles were identified in which four of these SNPs, including rs8028440, rs2289823, rs7403800, and rs3751566 posse greater prevalence due to their high-expression levels [27]. In this study, we investigated an identical genetic variation of rs8028440 polymorphism in CYFIP1, which to the best of our knowledge, has not been investigated prior. Based on our results, the probability of rs 8028440 polymorphism incidence was mainly related to the gender of patients and its occurrence in females was more than that of males. The P-value $(\mathrm{p}=0.106)$ and $\mathrm{OR}$ (0.69) analysis indicated that rs 8028440 polymorphism was not directly associated with any ASD occurrence under any of the examined genetic models. It has to be noted that our results are relative to a restricted population of subjects and generalization of these findings must be validated on a larger population.

The association between SNPs within several genes and the risk of ASD in different populations was determined. The association between SNP rs1009153 of CYFIP1, SNP rs7170637, and SNP rs3796863 of CD38 has been broadly investigated in Han Chinese [32], German [25], and American [33] populations of ASD and SCZ, respectively. Sayyad et.al in 2017 investigated the association between two functional polymorphisms (rs11639084 and rs4774388) in the retinoic acid-related orphan receptor-alpha (RORA) gene of Iranian subjects ASD through tetra primer-amplification refractory mutation system PCR. Based on their investigation results, no significant differences between SNP rs11639084 frequencies between patient and control groups were reported. However, the $\mathrm{T}$ allele frequencies and TT genotype of rs4774388 were significantly high in patients than in control subjects, which were associated with ASD risk in the dominant inheritance model. Ultimately, these findings have suggested the role of the RORA gene in the pathogenesis of ASD in the Iranian 
population [34]. Moreover, contribution of SNPs in the insulin receptor substrates including rs 1801123 of IRS1, and rs4773092 of IRS1/ IRS2 with ASD risk was carried out by Park et.al in 2016. Accordingly, direct sequencing of 180 Korean male ASD cases and 147 control cases indicated the involvement of rs1801123 in IRS1 and ASD in additive and dominant models. In addition, the association between rs1801123 and ASD was determined based on the allele frequency analysis [35].

ASD as a multifactorial disorder is mainly incorporated with both genetic variations and environmental factors and precise illumination of underlying molecular factors in ASD development along with providing novel comprehensive strategies for accurate and early detection of ASD is still the subject of most researches. In addition, providing a detailed map of genetic variations in ASD could enable early detection of high-risk patients for treatment and increasing their quality of life [36]. Recently, machine learning has been widely used to derive accurate predictive ASD model from autism databases [37]. Bioanalysis of ASD with respect to role of genetics could open new powerful windows to categorize the autism risk genes. Although, genetic information of ASD has been prosperously produced based on the massive genetic studies. However, these studies are costly and slow moving, highlighting the necessity of providing efficient approaches for identification of putative ASD risk genes. In this regard, Brueggeman et.al in 2020 tried to discover new ASD risk genes using genome-scale data and involvement of each gene in the etiology of ASD [38].

In conclusion, we conducted our research in Iranian ethnic population, to investigate the frequency of rs 8028440 polymorphism of CYFIP1 gene among 100 autism and 100 control cases. We found that the incidence of $\mathrm{C}$ alleles was more than the frequency of the T allele in healthy and ASD cases. Although, in the previous studies association between rs8028440 polymorphism of CYFIP1 gene was reported but our result was contradictory. Therefore, further investigations are necessary to find the role of CYFIP1 gene polymorphisms in the etiology of ASD along with identifying the predictor and comprehensive gene network in ASD cases.

\section{References}

1. Gmitrowicz A, Kucharska A. Developmental disorders in the fourth edition of the American classification: diagnostic and statistical manual of mental disorders (DSM IV -- optional book)]. Psychiatr Pol. 1994;28(5):509-21.

2. Freitag CM. The genetics of autistic disorders and its clinical relevance: a review of the literature. Mol Psychiatry. 2007;12(1):222.

3. Geschwind DH. Advances in autism. Annu Rev Med. 2009;60:367-380.

4. Belmonte MK, Cook EH Jr, Anderson GM, et al. Autism as a disorder of neural information processing: directions for research and targets for therapy. Mol Psychiatry. 2004;9(7):646663. Liu X, Takumi T. Genomic and genetic aspects of autism spectrum disorder. Biochem Biophys Res Commun. 2014;452(2):244-253.

5. Monaco AP, Bailey AJ. Autism. The search for susceptibility genes. Lancet. 2001;358 Suppl:S3.

6. Colvert E, Tick B, McEwen F, et al. Heritability of Autism Spectrum Disorder in a UK Population-Based Twin Sample. JAMA Psychiatry. 2015;72(5):415-423.
7. Tick B, Bolton P, Happé F, Rutter M, Rijsdijk F. Heritability of autism spectrum disorders: a meta-analysis of twin studies. J Child Psychol Psychiatry. 2016;57(5):585-595.

8. Belmonte MK, Cook EH Jr, Anderson GM, et al. Autism as a disorder of neural information processing: directions for research and targets for therapy. Mol Psychiatry. 2004;9(7):646663.

9. Cheng L, Ge Q, Xiao P, et al. Association study between BDNF gene polymorphisms and autism by three-dimensional gelbased microarray. Int J Mol Sci. 2009;10(6):2487-2500.

10. KimSJ, BruneCW, KistnerEO, etal. Transmission disequilibrium testing of the chromosome 15q11-q13 region in autism. Am J Med Genet B Neuropsychiatr Genet. 2008;147B(7):1116-1125.

11. Jiang YH, Yuen RK, Jin X, et al. Detection of clinically relevant genetic variants in autism spectrum disorder by whole-genome sequencing. Am J Hum Genet. 2013;93(2):249-263.

12. Bai $Y$, Xiang $X$, Liang $C$, Shi L. Regulating Rac in the nervous system: molecular function and disease implication of Rac GEFs and GAPs. Biomed Res Int. 2015;2015:632450.

13. Aguilar BJ, Zhu Y, Lu Q. Rho GTPases as therapeutic targets in Alzheimer's disease. Alzheimers Res Ther. 2017;9(1):97.

14. Huang GH, Sun ZL, Li HJ, Feng DF. Rho GTPase-activating proteins: Regulators of Rho GTPase activity in neuronal development and CNS diseases. Mol Cell Neurosci. 2017;80:1831.

15. Niftullayev S, Lamarche-Vane N. Regulators of Rho GTPases in the Nervous System: Molecular Implication in Axon Guidance and Neurological Disorders. Int J Mol Sci. 2019;20(6):1497.

16. Pathania M, Davenport EC, Muir J, Sheehan DF, LópezDoménech G, Kittler JT. The autism and schizophrenia associated gene CYFIP1 is critical for the maintenance of dendritic complexity and the stabilization of mature spines [published correction appears in Transl Psychiatry. 2014;4:e423]. Transl Psychiatry. 2014;4(3):e374.

17. Abekhoukh S, Bardoni B. CYFIP family proteins between autism and intellectual disability: links with Fragile X syndrome. Front Cell Neurosci. 2014;8:81.

18. Schenck A, Bardoni B, Moro A, Bagni C, Mandel JL. A highly conserved protein family interacting with the fragile X mental retardation protein (FMRP) and displaying selective interactions with FMRP-related proteins FXR1P and FXR2P. Proc Natl Acad Sci U S A. 2001;98(15):8844-8849.

19. Doornbos M, Sikkema-Raddatz B, Ruijvenkamp CA, et al. Nine patients with a microdeletion 15q11.2 between breakpoints 1 and 2 of the Prader-Willi critical region, possibly associated with behavioural disturbances. Eur J Med Genet. 2009;52(23):108-115.

20. van der Zwaag B, Staal WG, Hochstenbach R, et al. A cosegregating microduplication of chromosome $15 \mathrm{q} 11.2$ pinpoints two risk genes for autism spectrum disorder. Am J Med Genet B Neuropsychiatr Genet. 2010;153B(4):960-966.

21. Picinelli C, Lintas C, Piras IS, et al. Recurrent 15q11.2 BP1BP2 microdeletions and microduplications in the etiology of neurodevelopmental disorders. Am J Med Genet B Neuropsychiatr Genet. 2016;171(8):1088-1098.

22. Leblond CS, Heinrich J, Delorme R, et al. Genetic and functional analyses of SHANK2 mutations suggest a multiple hit model of autism spectrum disorders. PLoS Genet. 2012;8(2):e1002521.

23. Alvarez-Mora MI, Calvo Escalona R, Puig Navarro O, et al. Comprehensive molecular testing in patients with high functioning autism spectrum disorder. Mutat Res. 2016;784- 
785:46-52.

24. Waltes R, Duketis E, Knapp M, et al. Common variants in genes of the postsynaptic FMRP signalling pathway are risk factors for autism spectrum disorders. Hum Genet. 2014;133(6):781-792.

25. Toma C, Torrico B, Hervás A, et al. Exome sequencing in multiplex autism families suggests a major role for heterozygous truncating mutations. Mol Psychiatry. 2014;19(7):784-790.

26. Wang J, Tao Y, Song F, Sun Y, Ott J, Saffen D. Common Regulatory Variants of CYFIP1 Contribute to Susceptibility for Autism Spectrum Disorder (ASD) and Classical Autism. Ann Hum Genet. 2015;79(5):329-340.

27. Noroozi R, Omrani MD, Sayad A, Taheri M, Ghafouri-Fard S. Cytoplasmic FMRP interacting protein $1 / 2$ (CYFIP1/2) expression analysis in autism. Metab Brain Dis. 2018;33(4):13531358.

28. Loesch DZ, Godler DE, Khaniani M, et al. Linking the FMR1 alleles with small CGG expansions with neurodevelopmental disorders: preliminary data suggest an involvement of epigenetic mechanisms. Am J Med Genet A. 2009;149A(10):2306-2310.

29. Abekhoukh S, Sahin HB, Grossi M, et al. New insights into the regulatory function of CYFIP1 in the context of WAVE- and FMRP-containing complexes. Dis Model Mech. 2017;10(4):463474.

30. Magdalon J, Sánchez-Sánchez SM, Griesi-Oliveira K, Sertié AL. Dysfunctional mTORC1 Signaling: A Convergent Mechanism between Syndromic and Nonsyndromic Forms of Autism Spectrum Disorder?. Int J Mol Sci. 2017;18(3):659.

31. Zhao Q, Li T, Zhao X, et al. Rare CNVs and tag SNPs at 15 q11.2 are associated with schizophrenia in the Han Chinese population. Schizophr Bull. 2013;39(3):712-719.

32. Higashida H, Yokoyama S, Huang JJ, et al. Social memory, amnesia, and autism: brain oxytocin secretion is regulated by $\mathrm{NAD}+$ metabolites and single nucleotide polymorphisms of CD38. Neurochem Int. 2012;61(6):828-838.

33. Sayad A, Noroozi R, Omrani MD, Taheri M, Ghafouri-Fard S. Retinoic acid-related orphan receptor alpha (RORA) variants are associated with autism spectrum disorder. Metab Brain Dis. 2017;32(5):1595-1601.

34. Park HJ, Kim SK, Kang WS, et al. Association between IRS1 Gene Polymorphism and Autism Spectrum Disorder: A Pilot CaseControl Study in Korean Males. Int J Mol Sci. 2016;17(8):1227.

35. de la Torre-Ubieta L, Won H, Stein JL, Geschwind DH. Advancing the understanding of autism disease mechanisms through genetics. Nat Med. 2016;22(4):345-361.

36. Thabtah F. Machine learning in autistic spectrum disorder behavioral research: A review and ways forward. Inform Health Soc Care. 2019;44(3):278-297.

37. Brueggeman L, Koomar T, Michaelson JJ. Forecasting risk gene discovery in autism with machine learning and genome-scale data. Scientific reports. 2020;10(1):1-11. 